\title{
DERMATOLOGIC MISNOMERS
}

MOSES SCHOLTZ, M.D.

Instructor of Dermatology, College of Physicians and Surgeons, University of Southern California; Dermatologist to Graves' Dispensary, Los Angeles Medical Department., University of California, and to Kaspare Cohn Hospital

LOS ANGELES

That the words should carry the exact and definite meaning assigned to them, would seem to be a natural requisite and necessity for an intelligent and productive exchange of ideas. That a clear and definite terminology is a sine qua non in any branch of science, seems also to be a truism. Medicine, as a branch of natural sciences, dealing with a concrete and tangible subject matter, should be capable of an exact and definite terminology and should be particularly insistent on the correct use of it.

Of all branches of clinical medicine, dermatology, which enjoys a unique distinction of visualizing its subject matter and of being able to verify its clinical observations by the histopathologic study, would seem to be the one best capable to select terminology of a steady and definite content and to attain the complete unity between a dermatologic term and the clinical concept expressed by this term. Yet how often a medical reader is struck by a loosely constructed sentence, wrongly used terms and a vaguely conceived idea in dermatologic writings, particularly in the general medical press. However, the purpose of this paper is to call attention, not to the casual lapses and carelessness of individual writers, but to the wrong designations and terms sanctioned by the daily use of the body of dermatologists and incorporated in the official dermatologic nomenclature.

\section{PHILOLOGIC MISNOMERS}

Let it be understood, though, that the writer does not protest here against purely philologic misnomers. The bulk of the original dermatologic names have been handed to us by Greeks and Romans. These names notoriously misfit their respective dermatoses and do not convey in the least their most striking clinical and morphologic characteristics.

For instance, eczema means in Greek "boiling out," presumably, referring to its exudation and weeping. Obviously, it covers only one clinical feature of eczema, and does not fit at all for all classes of dry eczemas. Psoriasis means in Greek. "itch," another greatly misleading term. Itching is a casual, rather uncommon and the least characteristic 
feature of psoriasis. Erysipelas means in Greek, "red skin," obviously, a meaningless and misleading name, as it can be applied to any inflammatory dermatosis. Acne means "a pointy prominence," a vague term that can be applied to any papular eruption. Lichen means symbiotic algae and fungi - a rather mysterious and symbolic designation which gives no inkling as to its clinical characteristics. Sycosis, "fig like" in Greek, is another image symbol of doubtful success. Sarcoma, in Greek "flesh," is another vague term which can be applied to any raw looking lesion.

These are a few of the many philologic inconsistencies in dermatologic nomenclature. Glaring as they are from a philologic point of view, from a dermatologic point of view they are the least harmful, and can be left undisturbed. The mind of the modern clinician is too far removed from classic influences, and he is not aware of this offence against classical languages. The abuse of Greek terms does not interfere with his clear conception and visualization of the clinical pictures conveyed by these terms, and does not conflict with his reasoning and analysis of clinical and pathologic facts.

The writer is willing to overlook also philologic incongruities and disharmonies of coupling together the Greek and Latin words, such as erythema multiforme, sycosis vulgaris, pityriasis versicolor, etc.

\section{DESCRIPTIVE MORPHOLOGIC AND PATHOLOGIC MISNOMERS}

Far more important and unjustifiable from the dermatologist's point of view, are the names and designations which frankly misrepresent clinical and pathologic characteristics of the dermatoses to which they are attached. Many of these names are of historical interest only, as they represent the best dermatologic thought at the time of their creation. It is high time, however, to revise dermatologic nomenclature in the light of modern research.

It is surprising how little has been attempted by the American dermatologists to expurgate and modernize their nomenclature. The latest effort was made in 1914 by the Committee of Classification of the American Dermatological Association. The Committee suggested few changes of a purely academic character, such as: steatoma for atheroma, molluscum for molluscum contagiosum, favus for tinea favosa, trichophytosis for tinea trichophytosa and xanthelasma for xanthoma palpebrarum. More important and substantial suggestions have been brought out by the individual speakers in the following discussion, but no systematic and comprehensive changes have been suggested.

It would be of immense benefit to the dermatologist to take an inventory of his nomenclature, to rearrange it according to modern 
conceptions and to do away with a lot of relics of the past which are unnecessary and no more serviceable. The very fact of redundancy and superabundance of dermatologic nomenclature so often commented on and lamented about, can be utilized for the purpose of selection of more correct and better fitting names. The correct and rational selection of nosologic names is probably of greater importance in dermatology than in any other branch of clinical medicine, as dermatologic classification is still largely based on morphologic differences. Dermatology more than any other branch of medicine is engaged in word painting of clinical pictures in its terminology, and is trying to embody in the names of its nosologic forms as many clinical characteristics and features as possible.

For the same reason the correct nomenclature would also relieve immensely the burden of the general practitioner struggling with the pitfalls of dermatologic diagnosis, for he is largely, and excusably so, looking for morphologic characteristics in the names of dermatoses.

The writer wishes to submit here the most glaringly inconsistent and misleading misnomers of dermatologic nomenclature and pleads for their revision and correction.

\section{ERYTHEMA GROUP}

1. Erythema induratum.-Possibly, the most glaring dermatologic misnomers can be found in the group of erythemata. Erythema in the strict and correct meaning of this word is a well defined and clear cut clinical and pathologic entity, and means hyperemia, active or passive. Hence no condition which shows clinical phenomena of infiltration or exudation can be termed erythema. How untenable and undefensible then is the term erythema induratum! It is a pathologic impossibility and clinical self contradiction. It is self evident that the pathologic equivalent of erythema hyperemia cannot produce clinically either infiltration or ulcerations. Erythema induratum is admittedly a granuloma of tuberculous nature and should be properly classed with the tuberculous group of dermatoses. Tuberculoderma ulcerosum or Bazin's disease, as suggested by Pollitzer of the Classification Committee, in 1914, is a perfectly rational name and deserves an unqualified approval.

2. Erythema Nodulare.-This is an equally untenable name. What is in common, both clinically and pathologically, between a mere hyperemia and inflammatory tumor-like nodules of erythema nodulare? The lack of exact knowledge of erythema nodulare makes it difficult to select a rational name for it, but the present name is, certainly, too grossly misfit to be tolerated any longer. 
3. For the same reasons the generic term erythema multiforme is untenable, as among numerous variations only erythematous groups are true erythemata. The only variations, to which a true erythema is subject, are being àrterial or venous, acute or chronic. Evidently, erythema, as such, cannot change its form into a papule, nodule or bulla.

4. On the same grounds the term erythema hyperemicum used by many textbooks, should be discarded as being misleading and tautologic in nature. There is no other kind of erythema but hyperemic.

We cannot use the same word erythema, both as a pathologic term designating a specific and well defined condition of hyperemia and also as a generic name for multiform lesions of the skin. To do so means not only to bring an utmost confusion into dermatologic classification, but also to be guilty of grossest violation of the fundamental principles of scientific terminology. For want of a better name why not call erythema multiforme dermatosis multiforme? It is less specific, but it is also less objectionable.

\section{DERMATITIS GROUP}

The same error in using the name dermatitis in a double capacity, both as a specific pathologic term and as a generic term designating multiform inflammatory disorders of the skin, has led to a number of dermatologic misnomers. Dermatitis is a well defined clinical syndrome. In spite of the strenuous efforts by some clinicians to separate it, as an independent clinical entity, it is synonymous and to all intents and purposes identical with eczema. It always signifies a diffuse inflammation of the skin, erythematous, papular or vesicular in character, with ill defined borders, desquamating or weeping and infiltrated in the chronic stage, itchy and without any tendency to a cyclic or self limited course. Any skin disorder differing widely from this classical syndrome should not be regarded as dermatitis.

\section{DERMATITIS VS. ECZEMA}

From this point of view the following names call for a revision: 5. The name infectious eczematoid dernatitis, suggested in 1902 by Engman for eczema associated with staphylococci, can be challenged on two points. First, the term eczematoid dermatitis implies clinical or morphologic differences between eczema and dermatitis. Yet no tangible or demonstrable evidence, either clinical or pathological, has been advanced to sustain the duality of these two terms. This has been amply brought out in a thorough and comprehensive literary and pathologic study by Walter Highman in 1917. All attempts by various writers to force such duality has led to umatural and conflicting deductions. 
Let us consider, for instance, the differential claim that dermatitis is mostly of local and well ascertainable etiology, while eczema is of systemic and unknown origin. The very basis of this differentiation can hardly be sustained. The mere difference of etiology could warrant only a separation of idiopathic eczemas from specific eczemas and idiopathic dermatitides from specific dermatitides, but it does not justify the creation of two separate clinical entities to express merely the difference of etiology in otherwise identical conditions. Moreover, this claim admits most striking and conflicting exceptions. For instance, is dermatitis exfoliativa or dermatitis herpetiformis of local, easily ascertainable origin? On the other hand, so-called parasitic or trade eczemas are of local, readily ascertainable origin. The efforts to retain the two terms as independent entities have led to confusion and conflicting deductions even on the part of the most competent observers. Thus, discussing the relative intensity of the pathologic process of dermatitis and eczema in the chapter on eczema due to irritant plants, Pusey says: "These frequently produce a reaction so violent that it oversteps the bounds that we assign to eczema. Such cases are considered under various forms of dermatitis."

On the other hand, says Stelwagon: "All the agencies capable of bringing on by their local or weakening action erythema or dermatitis are also capable in such persons of provoking a veritable eczema, especially when long continued or repeatedly acting."

It seems that nothing but the force of habit and mental inertia guides the body of dermatologists in using the terms dermatitis and eczema. The same writers who insist on the differentiation of these terms use them indiscriminately and interchangeably in some cases, such as seborrheic eczema and seborrheic dermatitis, or trade eczema and trade dermatitis. The prevailing confusion is undoubtedly due to the insistence of differentiation of two identical and interchangeable clinical concepts. It occurs to the writer that the time honored but vague term eczema can be discarded and left for the laymen's use only. Dermatitis, as the more rational and scientific term, should be the only one used by dermatologists.

INFECTIOLS ECZEMATOID DERMATITIS VS. STREPTODERMATITIS

The name infectious eczematoid dermatitis is open to objections becat1se it emphasizes the difference between eczema and dermatitis which cannot be sustained. The other adjective-“infectious"-can also be challenged as being too comprehensive and to that extent misleading. The cases described by Engman in 1902 are due to staphylococci or possibly to mixed staphylococci and streptococci infection. 
The cases are closely allied, if not identical, with so-called dermatitis repens, and are aptly termed by Sabouraud exudative streptodermatitis. The specific bacteriologic etiology is the most important differential feature of these cases. Yet the name "infectious" is general in character and may mean any parasitic dermatitis, such as seborrheic, trychophitic, one due to scabies or to any other animal or vegetable parasite. Sabouraud's appellation streptodermatitis or staphylodermatitis seems to be more exact and appropriate, and deserves a decided preference.

6. In this connection it may be mentioned that the name dermatitis repens does not fit perfectly its clinical content. As cited above, it is closely allied, if not identical with the form described by Engman. While pathologically, it is a frankly inflammatory process, clinically it differs markedly from the ordinary clinical conception of dermatitis, as a diffuse catarrhal inflammation of the skin. Sharply defined serpiginous borders, advancing vesicular edges with an undermined detached epidermal collarette, glazy or shiny surface of the central parts, oozing or dry, occasionally slightly atrophic, healing over older central areas of the lesion. lack of infiltration in spite of the duration of many years, absence of itching and a decided preference in distribution for the extremities-all these features render it easily differentiated on clinical grounds from ordinary dermatitis or eczema. The adjective "repens," meaning creeping, is also slightly misleading. as the extension of the lesion through an advancing edge is characteristic of all parasitic conditions, be it seborrhea, lupus vulgaris, tinea, etc. Streptodermatitis or staphylodermatitis would seem to be a rational substitute, both for dermatitis repens and infectious eczematoid dermatitis.

7. Dermatitis papillaris capillitii clinically is anything but dermatitis. It is distinctly a follicular inflammatory process with secondary atrophy and keloidal sclerosis. The clinical and pathologic picture of the condition is so radically different from an ordinary dermatitis that it is almost difficult to account for the origin of the name dermatitis papillaris capillitii, which should be discarded as grossly defective and misleading. The names already suggested by various observers, such as acne keloid or folliculitis keloidalis, are much more rational and should be used instead.

8. Dermatitis factitia is another unwarranted name. The clinical picture of it practically never bears the slightest resemblance to the diffuse skin catarrh of dermatitis. In fact, a diagnosis of dermatitis factitia is made essentially on the presence of the clinical features absolutely exclusive of common dermatitis, such as discrete and sharply defined lesions, crusted, bullous or gangrenous in character, of pecu- 
liarly fancy or regular outline, suddenly cropping out on widely scattered parts of the body. A substitution of dermatosis factitia for dermatitis factitia would seem to be appropriate.

9. Dermatitis herpetiformis is a name given by Duhring to his brilliant clinical synthetic conception of papular, vesicular and bullous toxic dermatoses. One would naturally hesitate challenging the name selected by one of the giants of dermatology. However, analyzing the picture of dermatitis herpetiformis, it is hard to see the justification for the term dermatitis. The average case of dermatitis herpetiformis is so utterly unlike any dermatitis or eczema that none of the numerous American textbooks finds it necessary even to differentiate it from any form of dermatitis. It is true, the individual lesions and patches are inflammatory in character, but so are individual lesions of herpes, lichen, psoriasis, infectious exanthemas, etc.; yet we do not call them dermatitis, as a whole, because the composite clinical picture and the clinical behavior are so widely different from the common conception of dermatitis. A substitution of dermatosis herpetiformis or the simple name of Duhring's disease seems to be a nearer approach to the correct name.

10. Dermatitis venenata and dermatitis medicamentosa, jointly considered, call for some comment. Dermatitis venenata by itself is a correct term and represents a perfect type of dermatitis due to a local irritation. But the adjectives venenata and medicamentosa are synonymous, if not identical, and they do not express the assumed difference in meaning unless by an arbitrary mental reservation. Dermatitis medicamentosa expresses correctly the clinical type of lesion only in the erythematous group of drug rashes. But an equally large, if not a larger group of drug eruptions comprising bullous, carbuncular, gangrenous, herpetic, keratotic, nodular, pigmentary types, is, obviously, not covered by the term dermatitis. A more general and comprehensive name, dermatosis medicamentosa, seems to be more proper.

11. Lichen scrophulosorum is one of the names that calls loudly for a revision. Its clinical resemblance to the lichen type is so crude and remote as to be totally misleading. In fact, the only feature in common is the papule formation. Everything else - color, distribution, grouping, clinical course, surface and appearance of the lesions, subjective symptoms, etiology and pathology are entirely different. Since the general consensus of opinion regards lichen scrofulosorum as one of the tuberculids, it seems that follicular or papular tuberculid would be a more correct name.

Closely allied clinically to lichen scrofulosorum are acnitis and folliclis, which are open to objections on similar grounds, particularly the first. These names were, obviously, selected because of their clinical 
resemblance to acne and folliculitis. Speaking on general principles, the selection of names on the strength of similarity of one condition to another is permissible and, indeed, may be indispensable, when the condition under consideration is not sufficiently undersood to name it on its own characteristics. Such is not the case at the present time in regard to acnitis and folliclis. Both conditions are known to be tuberculous granulomata and by most of observers are considered to be mere variants of the same process. The name of papulonecrotic tuberculid would seem to be perfectly fitting and rational.

13. The same reasoning will hold good for acne varioloformis, provided it will prove to be a tuberculid. However, until Sabouratud's claim that it is due to staphylococcic infection on a seborrheic base will be refuted, the revision will be held in abeyance.

14. Acne rosacea is not an entirely satisfactory name and can be improved by dropping the appellation acne, which has already been done by several writers. It is true that acne and diffuse seborrhea are commonly associated with rosacea, as a secondary phenomenon, but the primary and essential pathologic process of rosacea - the loss of the vasomotor tonus, permanent congestive hyperemia and telangiectases - is of entirely different nature than that of acne or seborrhea. From a broader biologic point of view acne vulgaris and rosacea are quite opposite to each other in character. Acne vulgaris is mostly found in adolescents, and is the expression of physiologic exuberance and functional glandular hyperactivity. Rosacea affects mainly people after 30 or 40 , is a degenerative phenomenon and the expression of premature debility or senility of the skin. From this point of view the name acne rosacea is rather misleading and self contradictory. While the elements of acne and seborrhea must be recognized as a component part of the clinical picture of rosacea, they should not be overemphasized to the extent of incorporating them into the names. The plain name of rosacea is to be preferred.

15. One of the most striking illustrations of the callousness and indifference of dermatologists, as a body, to various monstrosities of dermatologic nomenclature is the retention and use by every textbook of the terms sycosis vulgaris and sycosis parasitica, in spite of the universal recognition of the gross imperfection and misleading nature of these terms. The conditions are both parasitic; one is due to staphylococci, the other to the trichophyton fungus. Why not use already available rational names - sycosis staphylogenes or coccogenes and sycosis trichophyton or hyphogenes?

16. Closely related to this is the time-honored name, ringworm. Who can deny the misleading and ensnaring influence of this name on the general practitioner who persistently refuses to diagnose a skin 
lesion as ringworm unless it looks like a ring? Yet in the total number of clinical forms of tinea due to the trichophyton and microsporon audouini fungus, ringlike lesions probably constitute the minority. A diagnosis of the average case of tinea is made clinically on sharply defined borders, superficial character of the lesions and the mode of development rather than on the annular form. Tinea trichophyton should be the name used, and ringworm should be reserved for the layman's use only.

17. In the name tinea versicolor the designation versicolor is rather inexact and misleading. An average case of tinea versicolor does not present any diversity of color, as the adjective implies. On the contrary, it has rather a homogeneous and uniform color of a fawnish yellow brown tinge, closely simulating chloasma. Tinea furfuracea microsporina would be a more rational name, even though it is less euphoneous. By the way, it may be remarked here that euphoneousness and other esthetic requirements can hardly be considered in the selection of a dermatologic name, and if they are, they may be considered only after all other requirements have been fully met.

In concluding the list of the most glaring misnomers we may say that the so often commented on confusing name of tubercular syphiloderma should be permanently abolished in favor of the English adjective already in use, nodular syphiloderma.

The above list of misnomers and suggested corrections is offered here without any claims of being final or comprehensive, merely to stimulate a discussion and to hasten the revision of dermatologic nomenclature which has already been unduly delayed. 